\title{
Clinical Effect of Ultrasonic Electrical Stimulation for Recent Pain After Minimally Invasive Interventional Therapy with Disc Degenerative Diseases
}

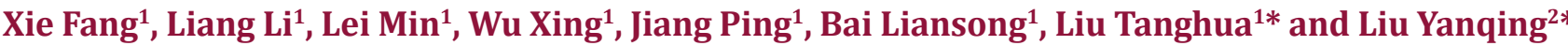 \\ ${ }^{1}$ Tongji Hospital of Lizhuang, Yibin, Sichuan, PR China \\ ${ }^{2}$ Beijing Tiantan Hospital Affiliated to Capital Medical University, Beijing, PR China \\ *Corresponding author: Liu yanqing, Beijing Tiantan Hospital Affiliated to Capital Medical University, Beijing, PR China
}

Liu Tanghua, Tongji Hospital of Lizhuang, Yibin, Sichuan, PR China

ARTICLE INFO
Received: 豐 February 14, 2020
Published: 慧 February 25, 2020

Citation: Xie Fang, Liang Li, Lei Min, Wu Xing, Jiang Ping, Bai Liansong, Liu Tanghua, Liu Yanqing. Clinical Effect of Ultrasonic Electrical Stimulation for Recent Pain After Minimally Invasive Interventional Therapy with Disc Degenerative Diseases. Biomed J Sci \& Tech Res 26(1)-2020. BJSTR. MS.ID.004280.

Keywords: After Interventional Therapy with DDD; The Recent Pain; Ultrasonic Electrical Stimulation

\begin{abstract}
Objective: To explore the curative effect of ultrasonic electrical stimulation for recent pain after minimally invasive interventional therapy for disc degenerative diseases (DDD).
\end{abstract}

Methods: From January to August 2019, 130 patients with DDD who were treated by minimally invasive intervention of intervertebral disc, and then were randomly divided into electrical stimulation group and conservative treatment group. After minimally invasive intervention, the electrical stimulation group was treated by ultrasonic electrical stimulation once a day for 7 days. The conservative treatment group was treated with conventional acupuncture and massage once a day for 3 days. The pain degree of before treatment, after treatment for 3 days, 7 days and 1 month were evaluated by pain numerical rating scale (NRS), the curative effect was evaluated by NRS weighting method.

Result: There was no significant difference in NRS score between the two groups before treatment $(P>0.05)$. NRS score in electrical stimulation group were significantly lower than these in conservative treatment group which were received conventional acupuncture and massage after treatment for 3 days, 7 days and one month $(P<0.001)$. The curative effect of electrical stimulation group on one month after interventional treatment was significantly higher than that of conservative treatment group $(P<0.001)$.

Conclusion: The therapeutic effect of ultrasonic electrical stimulation is better than that of traditional acupuncture and massage for recent pain after minimally invasive interventional therapy with DDD, it is worthy to popularize in clinical treatment.

\section{Short Communication}

Spinal degenerative radicular pain is a kind of disease characterized by pain in the innervated area of spinal cord. Clinically, the most common pain is neck, waist, back and limbs. According to statistics, the incidence of cervical spondylosis and low back pain is $3.8 \% \sim 17.6 \%$ and $67 \%$ of adults respectively, $56 \%$ of which are root pain or sciatica [1]. Minimally invasive interventional therapy (such as nerve root block, radiofrequency therapy, nerve regulation, spinal endoscopy, etc.) has a good effect for DDD [2], but it needs to recover one to three months after minimally invasive interventional therapy and tend to be stable. During this period, mechanical compression and inflammatory stimulation will lead to. Ultrasound electrical stimulation is an innovative physiotherapy for chronic pain repair, which integrates biological wave, bioelectricity, biomagnetism, negative oxygen ion and small molecule transdermal delivery technology. It has good therapeutic effect in pain treatment, tissue inflammation elimination and damaged nerve repair. Since there are still pain caused by inflammatory stimulation and other factors after minimally invasive interventional therapy, in order to effectively alleviate the pain in the recovery period after minimally invasive interventional therapy and improve the patients' 
satisfaction with the efficacy, the physical therapy was carried out by using supernatural electrical stimulation, and its short-term (within 7 days) and long-term (one month) effects were evaluated.

\section{Materials and Methods}

\section{General data}

A total of 130 patients with DDD including 47 males and 83 females, aged 26-85 years with an average age of (53.62 \pm 7.7$)$ years, were selected for minimally invasive interventional treatment of intervertebral disc in our hospital from January to August 2019. The lesions were including cervical spondylosis and degenerative disease of lumbar intervertebral disc. The patients were randomly divided into two groups by random number method, with 65 patients respectively in two groups.

\section{Methods}

The patients in two groups were treated with ultrasonic stimulation and conventional conservative therapy respectively after minimally invasive interventional therapy. In the electric stimulation group, the corresponding spinal nerve innervation area was treated with ultrasonic electric stimulation. The electric stimulation treatment was carried out with TU-B02 super generation electric stimulation treatment instrument (Shenzhen tairuikema Medical Technology Co., Ltd.,China) with current range at 40 to 50 , once a day, 20 min each time, and the treatment course was 7 days. The patients in conservative treatment group were treated with conventional acupuncture and massage once a day for 20 minutes for 3 consecutive days to assess the pain, and the patients in the conservative treatment group were scheduled for

Table 1: Comparison of general data between two groups [n (\%)]. outpatient follow-up and assessment one week and one month later.

\section{Observation Indexes}

Before treatment, 3 days, 7 days and 1 month after treatment, the pain degree of patients was evaluated by pain number rating scale (NRS). The evaluation of curative effect was carried out after the treatment one month by NRS weighting method. Curative effect : NRS score (a-b) / a $\times 100 \% \geq 75 \%$,excellent: NRS score (a-b) / a $\times$ $100 \% \geq 50 \% \sim 75 \%$, effective: NRS score (a-b) / a $\times 100 \% \geq 25 \% \sim$ $50 \%$, ineffective: NRS score (a-b) / a $\times 100 \%<25 \%$. Total effective rate $=($ cureative + excellent + effective $) /$ total number of patients.

a: NRS before treatment; b: NRS after treatment.

\section{Statistics}

Data were processed by SPSS 20.0 statistical software (SPSS software co., Chicago, IL, USA). Frequency or rate was used to describe the counting data, chi square test was used for the comparison between groups, rank sum test was used for the comparison of curative effect (grade data). The measurement data was described by mean \pm standard deviation $(\mathrm{M} \pm \mathrm{SD}$.) difference between groups was compared by independent $t$ test or one-way ANOVA (SNK method was used for the pairwise comparison), and the level of statistical significance was taken as $\mathrm{P}<0.05$.

\section{Result}

\section{General Data Comparison}

There was no significant difference in gender, age and lesion between the two groups $(\mathrm{P}>0.05)$ (Table 1$)$.

\begin{tabular}{|c|c|c|c|c|c|}
\hline \multirow{2}{*}{ General Data } & Items & Conservative Treatment Group & Electric Stimulation Group & $\chi^{2}$ & P \\
\hline \multirow{2}{*}{ Gender } & male & $26(40.0)$ & $21(32.3)$ & \multirow{2}{*}{0.833} \\
\cline { 2 - 5 } & female & $39(60.0)$ & $44(67.7)$ & 0.465 \\
\hline \multirow{2}{*}{ Age (years) } & $<50$ & $14(21.5)$ & $11(16.9)$ & \multirow{2}{*}{0.446} \\
\cline { 2 - 5 } & $\geq 50$ & $51(78.5)$ & $54(83.1)$ & 0.657 \\
\hline \multirow{2}{*}{ Lesion } & cervical vertebra & $11(16.9)$ & $9(13.8)$ & \multirow{2}{*}{0.836} \\
\cline { 2 - 5 } & lumbar vertebra & $54(83.1)$ & $56(83.2)$ & \\
\hline
\end{tabular}

\section{Comparison of NRS Between the Two Groups at Different Time Points of Post-Cure}

There was no significant difference of NRS between the two groups before treatment $(P>0.05)$. The significant difference of NRS were found between the two groups at 3 days, 7 days and 1 month of after treatment. The NRS of the electric stimulation group were lower than those of the conservative treatment group $(\mathrm{P}<$ 0.001). There were significant differences of NRS between the two groups at different time points of post-cure $(\mathrm{P}<0.001)$ (Figure 1$)$.

\section{Comparison of Analgesic Effect Grade Between the Two Groups on Post-Cure 1Month (m)}

The total effective rate of the conservative treatment group and electric stimulation group was $72.3 \%$ and $95.4 \%$ respectively. The electric stimulation group was superior to the conservative treatment group $(\chi 2=12.779, \mathrm{P}=0.001)$. There was also a statistically significant difference of analgesic effect grade between the two groups on post-cure 1 month $(\mathrm{m}),(\mathrm{P}<0.001)$ (Figure 2$)$. 


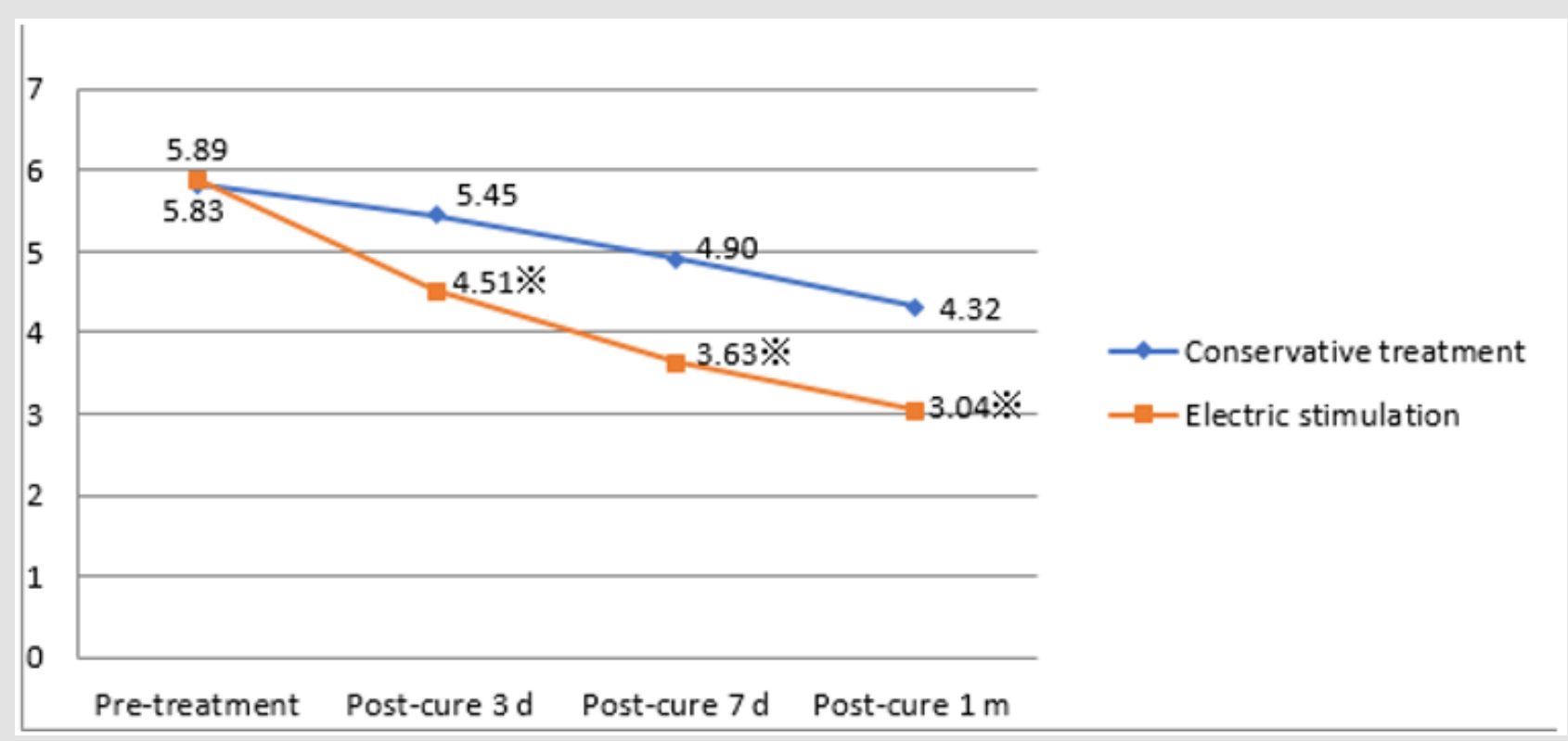

Figure 1: Comparison of NRS between two groups at different time points of post-cure $(x \pm s d)$.

Note: $※$ indicates compared with the conservative treatment group, $\mathrm{P}<0.001$.

\section{Conservative treatment Electic stimulation}

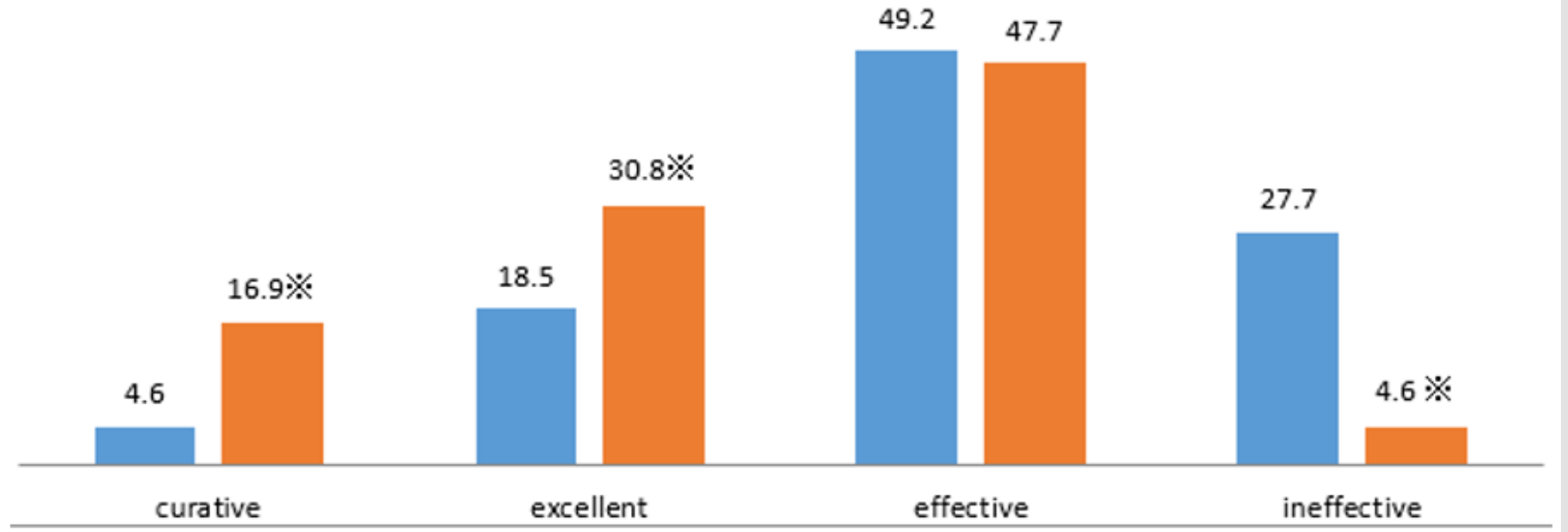

Figure 1: Comparison of analgesic effect grade between two groups on post-cure $1 \mathrm{~m}(\%)$.

Note: $\%$ indicates compared with the conservative treatment group, $\mathrm{P}<0.001$.

\section{Discussion}

Spinal degenerative radicular pain is a kind of disease characterized by pain in the innervated area of spinal cord caused by the invasion of nerve roots. The degradation of extracellular collagen and proteoglycan, the disorder of cell metabolism, and the degeneration of intervertebral disc vary in different degrees with the increase of age [3]. Timely and effective treatment is the key to recovery. At present, with the rapid development of minimally invasive interventional therapy technology, it is widely used in various medical fields [4,5], not only has the advantages of small trauma, high precision, good efficacy and easy recovery, but also can significantly improve the quality of life of patients [6]. However , some potential pain has always been a common problem for patients after minimally invasive treatment. In the recovery period of 1-3 months after interventional therapy, different treatment methods have different control effects for the pain, while electrical stimulation therapy has a good effect on postoperative pain $[7,8]$. 
Ultrasonic electrical stimulation therapy is an innovative chronic pain repair and treatment measure that integrates biological wave, bioelectricity, biomagnetism and small molecule transdermal delivery technology. It not only can effectively alleviate the pain caused by inflammation of myofascial, muscle and nerve root, but it also has repairing effect with nerve of damage by superposition of several technical principles of low medium frequency electricity, ultrasonic, electromagnetic and laser [9]. This study showed that the NRS of 3 days, 7 days and 1 month after minimally invasive interventional therapy were better than those of the conservative treatment group.

Electrical stimulation therapy could relieve the after-treatment pain, increase the comfort and improve the satisfaction of patients. The carrier bioelectricity of ultrasonic electric stimulation therapy instrument can regulate the membrane potential of neuron cells, promote the exchange of oxygen and nutrients of cells, so as to achieve the effect of repairing damaged nerves and removing inflammation. One month after treatment, the curative effect of the two groups was evaluated and found that the total effective rate of the electric stimulation group was significantly higher than that of the conservative treatment group $(95.4 \%$ vs $72.3 \%, \mathrm{P}=0.001$ ), indicating that the electric stimulation group was superior to the traditional conservative treatment in control recent pain effect after interventional treatment. In short, ultrasonic electrical stimulation can effectively relieve recent pain after interventional therapy for patients with DDD, and achieve relatively satisfactory results, which is of great significance to improve the comfort and quality of life of patients, so it is worthy to popularize in clinical treatment.

\section{ISSN: 2574-1241}

DOI: $10.26717 / B J S T R .2020 .26 .004280$

Liu Tanghua, Liu Yanqing. Biomed J Sci \& Tech Res

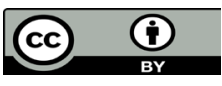

This work is licensed under Creative Commons Attribution 4.0 License

Submission Link: https://biomedres.us/submit-manuscript.php

\section{Disclosures/Conflict of interest}

The authors declare that there is no conflict of interest.

\section{References}

1. (2019) Pain branch, Chinese Medical Association. Expert consensus on the treatment of spinal degenerative radicular pain (China). J Natl Med J China 99(15): 1133-1137.

2. Türk C, Petř́ík A, Sarica K, Seitz C, Skolarikos A, et al. (2016) EAU Guidelines on Interventional Treatment for Urolithiasis. J Eur Urol 69(3): 475-482.

3. Genevay S, Courvoisier DS, Konstantinou K, Kovacs FM, Marty M, et al (2017) Clinical classification criteria for radicular pain caused by lumbar disc herniation: the radicular pain caused by disc herniation (RAPIDH) criteria. Spine J 17(10): 1464-1471.

4. Guevara-López U, Covarrubias-Gómez A, Elías-Dib J, Reyes-Sánchez A, Rodríguez-Reyna TS, et al. (2011) Practice guidelines for the management of low back pain. Consensus Group of Practice Parameters to Manage Low Back Pain. Cir Cir 79(3): 264-279, 286-302.

5. McGowan JE, Ricks CB, Kanter AS (2017) Minimally Invasive Treatment of Spine Trauma. Neurosurg Clin N Am 28(1): 157-162.

6. Koban O, Ogrenci A, Yaman O, Sedat Dalbayrak (2019) Phantom Radicular Pain Treated with Lumbar Microdiscectomy: A Case Report. Turk Neurosurg 29(1): 145-147.

7. Sadala AY, Machado AFP, Liebano RE (2018) Effects of transcutaneous electrical nerve stimulation on pain intensity during application of carboxytherapy in patients with cellulite: A randomized placebocontrolled trial[J]. J Cosmet Dermatol 17(6): 1175-1181.

8. Rock JM, Rainey CE (2014) Treatment of nonspecific thoracic spine pain with trigger point dry needling and intramuscular electrical stimulation: A case series. Int J Sports Phys Ther 9(5): 699-711.

9. Cressman JR, Gertz M, Chitnis PV (2017) Ultrasonic stimulation and metabolic stress in neuronal systems. J Acoust Soc Am 142: 2668.

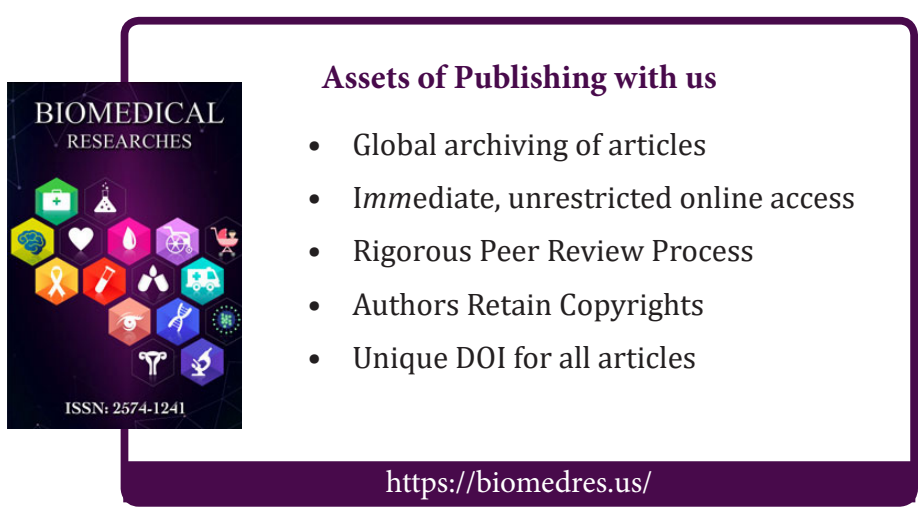

